\title{
A Personalized Collaborative Recommendation Algorithm Based on Hybrid Information
}

\author{
Dejia Zhang \\ Wenzhou Vocational and Technical College, Wenzhou 325035, China \\ zhangdejiaei@sina.com
}

Keywords: collaborative filtering, personalized, hybrid information, algorithm.

\begin{abstract}
Personalized recommendation systems can help people to find interesting things and they are widely used with the development of electronic commerce. Many recommendation systems employ the collaborative filtering technology, which has been proved to be one of the most successful techniques in recommender systems in recent years. With the gradual increase of users and items in electronic commerce systems, the time consuming nearest neighbor collaborative filtering search of the target customer in the total user space resulted in the failure of ensuring the real time requirement of recommender system. The paper proposed a personalized recommendation algorithm using hybrid information. User and item information is used for collaborative filtering to produce the recommendations. The recommendation joining user information and item information collaborative filtering is more scalable than the traditional one.
\end{abstract}

\section{Introduction}

The recommendation system in the electronic commerce website obtains the information from the customer, obtains the customer's interest, the hobby and the recommendation related commodity to the customer. Because the recommended system has great potential, so the research on the recommendation system is more and more. Collaborative filtering algorithm is one of the earliest and most successful recommendation techniques.

At present, the algorithm is successful in both practice and research. However, with the deepening of the application and the wide range of applications, it also exposes some points, the most important is the following two points. The algorithm can be used in real time. Search for thousands of "neighbors and target customers with similar interests, backgrounds of customers" but the huge online customer groups, to find the speed of the neighbors to be improved, and thus improve the scalability of the algorithm. The recommended quality of the recommendation system is as easy to make two kinds of errors as other query system. However, the recommended system is not recommended for the mistake, that is, the customer does not like the item recommendation system is recommended. In electronic commerce, we should try to avoid this kind of mistake. Because, the error will cause the customer's dissatisfaction, thus destroys the recommendation system's authority.

With the continuous expansion of the scale of electronic commerce system, the number of customers and the number of goods has increased dramatically, resulting in the calculation of the target user's nearest neighbor is not accurate, thereby reducing the system's recommendation quality. The paper proposed a personalized recommendation algorithm using hybrid information. User and item information is used for collaborative filtering to produce the recommendations. The recommendation joining user information and item information collaborative filtering is more scalable than the traditional one. 


\section{Problem analysis}

Collaborative filtering is the key problem to find the nearest neighbor, this step is the key to the whole process. And to find the best neighbor, we have to effectively build a good user - project score matrix. On the other hand, as customers, the continuous growth of goods, resulting in the user rating data is extremely sparse. The results are not recommended for certain customers and thus affect the accuracy of the recommendation.

Based on the product features, the collaborative filtering recommendation is proposed to construct the product file, and the potential of the user to a certain kind of product is based on the user's purchase quantity and the average purchase amount. Because of the construction of collaborative filtering user - project matrix. It is generally based on the user's score for all projects, as shown in figure 1 .

\begin{tabular}{|c|c|c|c|c|c|c|}
\hline \multirow{2}{*}{$\begin{array}{c}\text { Rating } \\
\text { User }\end{array}$} & \multicolumn{3}{|c|}{ Books } & \multicolumn{3}{c|}{ Videos } \\
\cline { 2 - 7 } & Book1 & Book2 & Book3 & Video1 & Video2 & Video3 \\
\hline User1 & 3 & 4 & 4 & 4 & 2 & 2 \\
\hline User2 & 1 & 4 & 3 & 2 & 1 & 1 \\
\hline User3 & 3 & 1 & 1 & 1 & 3 & 3 \\
\hline User4 & 2 & 3 & 3 & 2 & 2 & 2 \\
\hline User5 & 3 & 4 & $?$ & 2 & 1 & $?$ \\
\hline
\end{tabular}

Figure 1 An example

For example, you can consider in this regard, the project will be divided into different categories. Users in the same category of projects are more similar than in different types of projects more accurate. And it can also reduce the data's high dimension. Finally, the user's interest in each part of the project is calculated with a certain heuristic rule to merge each class of recommendation results as the user's final recommendation.

\section{Personalized collaborative recommendation algorithm based on hybrid information}

\section{The model of the method}

Collaborative filtering is one of the methods used in e-commerce recommendation system. Its core idea is that users tend to buy the goods which have similar interests. The basic starting point is that the customer is able to classify the customers. These three points constitute the basis of collaborative filtering, the more the user interest in the product, the higher the evaluation, as shown in figure 2. 


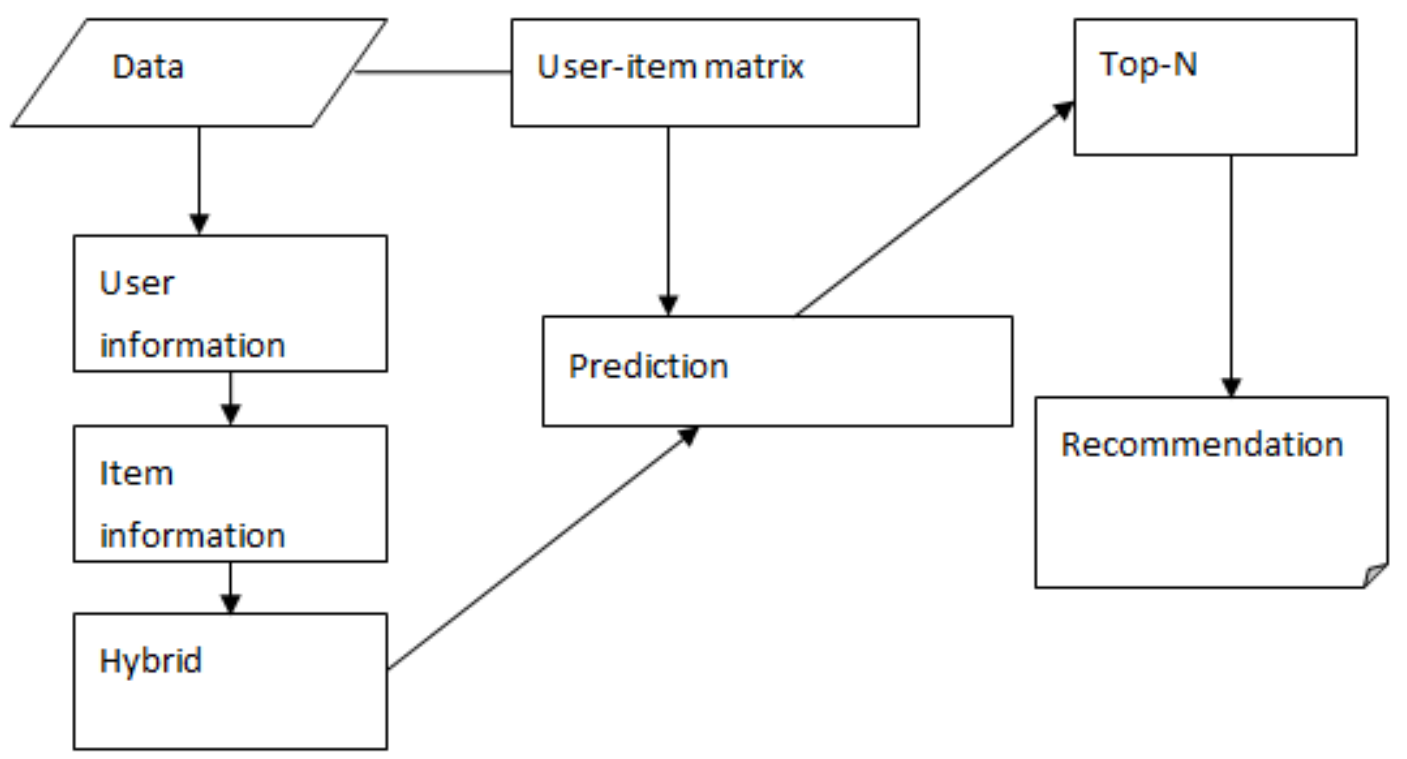

Figure 2 The model of the method

As a result of a lack of customer product rating data and customers can not accurately score, on the other hand, with the continuous growth of customers, goods, resulting in the user rating data is extremely sparse, the results are not recommended for some customers and thus affect the recommendation accuracy.

\section{The recommendation algorithm}

With the rapid growth and wide application of the Internet and Electronic Commerce systems, information system has provided an unprecedented abundance of information resources, and it has also led to the problem of information overload. Thus, methods to help find resources of interest have attracted much attention from both researchers and vendors. To deal with the problem, the personalized recommendation systems play a more important role. The famous electronic commerce website Amazon and CD-Now have employed recommender technique to recommend products to customers and it has improved quality and efficiency of their services.

It is based on the assumption that the ratings of the items are similar to those of the users. If most users have similar items on the basis of such a hypothesis: if the majority of users have similar ratings to some items, the current user ratings are similar to those projects. The current user ratings of the items are similar. Based on Project Nearest neighbor of the recommended system, the collaborative filtering recommendation algorithm is based on the similarity between the project score and the clustering center, which is based on the similarity between the target and the target, which is based on the similarity between the target items and the nearest neighbor of the target project, and the nearest neighbor of the target project is selected, and the nearest neighbor of the target is selected., all need to measure the project The similarity between the user rating data is shown below.

$$
A=\left[\begin{array}{l}
R_{1,1} \cdots R_{1, j} \cdots R_{1, n} \\
\cdots \cdots \cdots \cdots \cdots \cdots \cdots \\
R_{i, 1} \cdots R_{i, j} \cdots R_{i, n} \\
\cdots \cdots \cdots \cdots \cdots \cdots \cdots \\
R_{m, 1} \cdots R_{m, j} \cdots R_{m, n}
\end{array}\right]
$$

The method of measuring the similarity between I and project $\mathrm{J}$ is as follows. First, all users of project I and project $\mathrm{J}$ are obtained. Then, the similarity between I and $\mathrm{j}$ is calculated by different methods of similarity measurement.

1 cosine similarity: the project score as a vector of $m$ dimensional user space, if the user does not have a score for the project, the project's score is 0 , and the similarity between the items is measured by the cosine angle between vectors: 


$$
\operatorname{sim}(z l p, g s j)=\cos (\overrightarrow{z l p}, \overrightarrow{g s j})=\frac{\overrightarrow{z l p}, \overrightarrow{g s j}}{\|\overrightarrow{z l p}\| *\|\overrightarrow{g s j}\|}
$$

2 related similarity: the ij $U$ is expressed as a for the $I$ and $j$, and the SIM (J, I) of the I and $\mathrm{j}$ is measured by Pearson:

$$
\operatorname{sim}(i, j)=\frac{\sum_{c \in u_{i j}}\left(R_{c, i}-\overline{R_{i}}\right)\left(R_{c, j}-\overline{R_{j}}\right)}{\sum_{c \in u_{i j}}\left(R_{c, i}-R_{i}\right)^{2} \sqrt{\sum_{c \in u_{i j}}\left(R_{c, j}-R_{j}\right)^{2}}}
$$

3 modified cosine similarity: in the cosine similarity measure method does not consider the different user's rating scale problem, the modified cosine similarity measure method by subtracting the user's average score of the project to improve the above defects:

$$
\operatorname{sim}(i, j)=\frac{\sum_{c \in u_{i j}}\left(R_{c, i}-\overline{R_{i}}\right)\left(R_{c, j}-\overline{R_{j}}\right)}{\sqrt{\sum_{c \in u_{i j}}\left(R_{c, i}-R_{i}\right)^{2}} \sqrt{\sum_{c \in u_{i j}}\left(R_{c, j}-R_{j}\right)^{2}}}
$$

By the method proposed in this paper to get the nearest neighbor of the target project, the next step is to produce the corresponding recommendation.

$$
P_{u, T, I}=\overline{R_{T I}}+\frac{\sum_{n \in N N_{T I}} \operatorname{sim}(T I, n) *\left(R_{u, n}-R_{n}\right)}{\sum_{n \in N N_{T I}}(|\operatorname{sim}(T I, n)|)}
$$

\section{Summary}

Personalized recommendation systems can help people to find interesting things and they are widely used with the development of electronic commerce. Many recommendation systems employ the collaborative filtering technology, which has been proved to be one of the most successful techniques in recommender systems in recent years. With the gradual increase of users and items in electronic commerce systems, the time consuming nearest neighbor collaborative filtering search of the target customer in the total user space resulted in the failure of ensuring the real time requirement of recommender system. The paper proposed a personalized recommendation algorithm using hybrid information. User and item information is used for collaborative filtering to produce the recommendations. The recommendation joining user information and item information collaborative filtering is more scalable than the traditional one.

\section{Acknowledgement}

A Project Supported by Scientific Research Fund of Zhejiang Provincial Education Department.

\section{References}

[1] Gong S, Wang Y. Research on Security Strategy of Electronic Commerce Industry Websites, 2015 International Conference on Advances in Mechanical Engineering and Industrial Informatics. Atlantis Press, 2015.

[2] Gong S. Research on the Generated Path of Electronic Commerce System Based on Innovative High-skilled Technology, 2015 International Industrial Informatics and Computer Engineering Conference. Atlantis Press, 2015. 
[3] Songjie Gong, Liping Zeng, The Solution of Safety of Electronic Cash in E-Commerce under Cloud Computing Environment, Advanced Materials Research, Vol. 989, pp: 4314-4317, 2014.

[4] Xue, G., Lin, C., \& Yang, Q., et al. Scalable collaborative filtering using cluster-based smoothing. In Proceedings of the ACM SIGIR Conference 2005 pp.114-121.

[5] D. Bridge and J. Kelleher, Experiments in sparsity reduction: Using clustering in collaborative recommenders, in Procs. of the Thirteenth Irish Conference on Artificial Intelligence and Cognitive Science, pp. 144-149. Springer, 2002.

[6] B. Sarwar, G. Karypis, J. Konstan and J. Riedl, Recommender systems for large-scale e-commerce: Scalableneighborhood formation using clustering, Proceedings of the Fifth International Conference on Computer andInformation Technology, 2002

[7] Songjie Gong, Research on the Growth Mechanism of High-Skilled System in Computer Science and Technology, Applied Mechanics and Materials, Vol. 513, pp: 2748-2751, 2014. 